\title{
Re-evaluation of the phenotype caused by the common MATR3 p.Ser85Cys mutation in a new family
}

\section{Palmio, Johanna}

2016-04

Palmio , J , Evilä , A, Bashir , A, Norwood , F , Viitaniemi , K, Vihola , A, Huovinen , S , Straub , V , Hackman , P , Hirano , M , Bushby , K \& Udd, B 2016 , ' Re-evaluation of the phenotype caused by the common MATR3 p.Ser85Cys mutation in a new family ' , Journal of Neurology, Neurosurgery and Psychiatry , vol. 87 , no. 4 , pp. 448-450 . https://doi.org/10.1136/jnnp-2014-309349

http://hdl.handle.net/10138/161264

https://doi.org/10.1136/jnnp-2014-309349

publishedVersion

Downloaded from Helda, University of Helsinki institutional repository.

This is an electronic reprint of the original article.

This reprint may differ from the original in pagination and typographic detail.

Please cite the original version. 


\section{Re-evaluation of the phenotype caused by the common MATR3 p.Ser85Cys mutation in a new family}

\section{INTRODUCTION}

Late-onset autosomal dominant vocal cord and pharyngeal distal myopathy (VCPDM) was first described in an American family. ${ }^{1}$ A p.Ser85Cys mutation in MATR3 gene was later identified in this family, and also in one Bulgarian family and very recently, in seven families-six German and one Asian. $^{2-4}$ Muscle pathology of VCPDM was dominated by rimmed-vacuolar fibre degeneration. ${ }^{1} 2$ This year, two other mutations in MATR3, p.Phe115Cys and p.Thr622Ala, have been reported to cause autosomal dominant amyotrophic lateral sclerosis (ALS). ${ }^{5}$ The original American p.Ser85Cys mutated family was re-evaluated and the authors stated: "These clinical findings supported reclassification of this condition as slowly progressive ALS, and the presence of upper motor neuron signs in the form of brisk reflexes ruled out myopathy as the only cause of disease in this family." 5

We report a new family with the p. Ser85Cys mutation in MATR3, in which muscle atrophy and weakness are caused by progressive degenerative myopathy without any evidence of lower motor neuron defects.

\section{METHODS}

We investigated an American family with four affected siblings. Patients II.1, II.2 and II. 4 were more extensively examined and followed for 7, 9 and 8 years, respectively. The father and one of his brothers were affected (figure 1A). Muscle MRI was performed in the proband (II.2) and in the younger brother (II.4). Muscle biopsies were obtained from three patients and examined by standard histochemical and immunohistochemical stainings with antibodies for Matrin 3, TDP-43, p62, SMI-31 and myosin A4.74, slow (MHCs), neonatal (MHCn) and developmental (MHCd) isoforms. Immunofluorescence analysis was performed with conventional methods using antibodies for G3BP and TIA1 C-terminus. Matrin 3 distribution was analysed from soluble cytoplasmic and nuclear fractions using western blotting. 
Blood samples of all siblings in generation II were obtained and DNA was extracted by standard methods. Whole exome sequencing was performed on patient II.2 at ATLAS Biolabs $\mathrm{GmbH}$ using SeqCap EZ Human Exome Library V.2.0 (Roche NimbleGen), and captured DNA was sequenced on Illumina HiSeq 2000 platform, $2 \times 100 \mathrm{bp}$. Reads were processed following the Genome Analysis Toolkit best practices pipeline and the Human Gene Mutation Database was used to detect previously reported pathogenic mutations. Sanger sequencing was performed to confirm the findings.

\section{RESULTS}

The age of onset ranged from 31 to 48 years, and the first manifestation was either weakness in the hands or legs causing difficulty in dexterity, or standing on heels and toes. The weakness slowly progressed to proximal upper and lower limb muscles. Wasting of the small hand muscles was evident followed by atrophy in the distal lower leg and forearm (figure 1B). Waddling and steppage gait was observed. Fasciculations were absent in all siblings. In two patients, brisk reflexes without clonus or spread were noted; there were no definite pathological pyramidal tract reflexes (Hoffman, crossed adductors, Babinski sign). Several years after disease onset, two patients had mild to moderate hypophonia and dysphagia. Two patients did not have respiratory symptoms; the other two had dyspnoea on exertion, reduced forced vital capacity $(50 \%)$, or both.

Creatine kinase (CK) levels were normal or marginally elevated. Electromyography studies of all patients were consistent with myopathic changes. Fasciculations were absent. No chronic neurogenic changes (eg, large amplitude or long duration motor unit potentials) were observed in any of the studies. MRI showed marked fatty degeneration of dystrophic type in soleus and medial gastrocnemius in both patients studied (figure 1C). Less severe changes were observed in the anterolateral lower leg compartment and hamstrings in II.4. In muscle biopsy taken from the left tibialis anterior (II.2), there were numerous rimmed-vacuolated fibres (figure 1D). MyHC double staining showed a normal fibre type distribution without grouping. MHCd labelled most of the highly atrophic fibres and these were reactive for MHCn, although with less intensity in the moderately atrophic fibres and not in MHCd negative fibres. Matrin 3 immunohistochemistry showed a loss of protein in the central parts of myonuclei (figure 1D). Myofibrillar myopathy markers myotilin, desmin, $\alpha \mathrm{B}$-crystallin, and ectopic cytoplasmic dystrophin were negative, whereas TDP-43, p62, and SMI-31 accumulated in rimmed-vacuolar fibres (data not shown). Electron microscopy showed highly convoluted myonuclei. In immunofluorescent analysis, the TDP-43 binding partner TIA1 and TIA1 ligand G3BP showed increased expression. Matrin 3 analysed by western blotting showed no abnormalities compared to control (not shown).
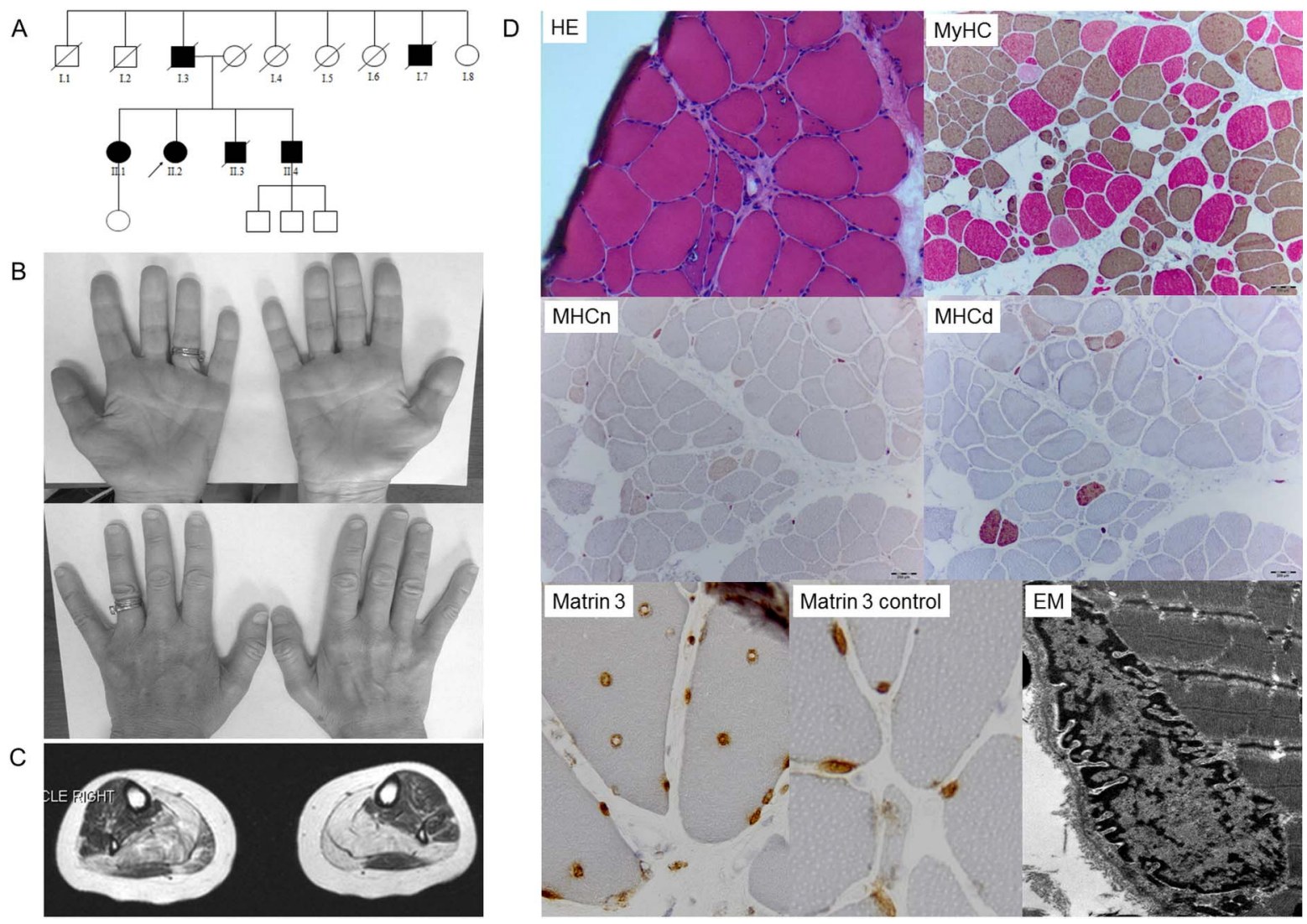

Figure 1 Pedigree and muscle findings of the family. Pedigree of the family (A). Wasting of the first dorsal interossei and thenar muscle of patient II.2 is seen on the right side (B). T1-weighted axial MRI scans of the lower legs of patient II.2 show prominent fatty degenerative changes in the soleus and medial gastrocnemius selectively sparing the lateral gastrocnemius and without significant changes in the anterior compartment (C). $\mathrm{H} \& \mathrm{E}$ stain section shows fibres with rimmed-vacuolar changes. Myosin heavy chain (MyHC) double staining with normal fibre type distribution, variation in fibre size ranging from highly atrophic rounded fibres to hypertrophic fibres, and numerous mostly rounded atrophic fibres of both type 1 (brown) and type Ila (red). Myosin heavy chain neonatal (MHCn) and developmental (MHCd) antibody reactivity in highly atrophic fibres and in moderately atrophic fibres. In internalised nuclei the central part showed depletion of Matrin 3 immunostaining with strong irregular label in the nuclear membrane. Matrin 3 normal control muscle showed a uniform reactivity in all myonuclei. Electron micrograph (EM) of the abnormally convoluted nuclear membrane. 
Exome sequencing revealed a known mutation in MATR3: c. $254 \mathrm{C}>\mathrm{G}$, p. Ser85Cys (NM_199189.2). ${ }^{2}$ The mutation was Sanger sequenced and was present in all four of the affected siblings.

\section{DISCUSSION}

We report a new VCPDM family with the p.Ser85Cys mutation in MATR3 showing less bulbar involvement, but respiratory failure in some cases. MATR3 as a cause of ALS was initially reported by Johnson et $a l^{5}$ based on the identification of segregating mutations in several families and subsequently replicated in exome data derived from an independent cohort of patients diagnosed with familial ALS. ${ }^{6}$ The pattern of combined features of ALS and myopathy has been recognised with other ALS-causing genes such as CHCHD10, VCP, hnRNPA1 and hnRNPA2B1. ${ }^{7-11}$ An assessment for a potential motor neuron component in our family showed brisk reflexes raising the possibility of minor upper motor neuron pathology, but there were no lower motor neuron abnormalities to account for the muscle atrophy.

Late-onset distal weakness with vocal cord and pharyngeal involvement has been the clinical hallmark of VCPDM. ${ }^{1}{ }^{2}$ However, bulbar symptoms can be absent in the beginning, as observed in our patients, and in a report of 16 German patients. $^{3}$ Respiratory insufficiency was evident in half of our siblings. The phenotype with this mutation is variable and the known hallmarks of the disease, dysphagia and dysphonia, can be subtle or absent. The distinct wasting of the first dorsal interossei and thenar muscles, with relative sparing of the hypothenar, was present in our patients, but never associated with clinical or electrophysiological fasciculations in muscles of the hands or elsewhere. Furthermore, motor unit potentials (MUPs) did not show increased duration or amplitude indicative of a chronic neurogenic process, but were clearly decreased in size indicating a myopathic process. Similarly, no changes compatible with a neurogenic process were observed on muscle biopsy. More than $10 \%$ of fibres expressed $\mathrm{MHCd}$, in contrast to neurogenic atrophy where MHCn reactive atrophic fibres are far more frequent than MHCd reactive fibres. We, thus, conclude that with the MATR3 p.Ser85Cys mutation in our family, the major process causing muscle atrophy and functional limitations is myopathic loss of muscle tissue.

Matrin 3 is a component of the nuclear matrix and has been associated with splicing and DNA replication. ${ }^{12}$ The abnormal nuclear distribution of Matrin 3 in immunohistochemistry with normal result in western blotting suggests that the disease pathomechanisms do not involve quantitative changes in Matrin 3 expression, but rather mislocalisation of the protein. Matrin 3 interacts with TDP-43, a protein linked to ALS/frontotemporal dementia. TDP-43 and other autophagic markers, p62 and SMI-31, were components of the rimmed-vacuolar pathology in our patients. Increase of TIA1 reactive cytoplasmic granules and increased G3BP labelling suggest they are involved. G3BP is a key component of stress granules and a ligand of Matrin 3, which may contribute to the phenotype similarity with Welander distal myopathy.

The cases reported here show that distinguishing between myogenic and neurogenic pathomechanisms requires careful assessment of clinical, electrophysiological, muscle morphological and muscle MRI techniques.

\section{Johanna Palmio, ${ }^{1}$ Anni Evilä, ${ }^{2}$ Ayat Bashir, ${ }^{3}$ Fiona Norwood, ${ }^{4}$ Kati Viitaniemi, ${ }^{1}$ Anna Vihola, ${ }^{2}$ Sanna Huovinen, ${ }^{5}$ Volker Straub, ${ }^{6}$ Peter Hackman, ${ }^{2}$ Michio Hirano, ${ }^{7}$ Kate Bushby, ${ }^{6}$ Bjarne Udd ${ }^{1,2,8}$}

${ }^{1}$ Department of Neurology, Neuromuscular Research Centre, Tampere University and University Hospital, Tampere, Finland

${ }^{2}$ Folkhälsan Institute of Genetics and the Department of Medical Genetics, Haartman Institute, University of Helsinki, Helsinki, Finland

${ }^{3}$ Newcastle University, Newcastle upon Tyne, UK ${ }^{4}$ Department of Neurology, King's College Hospital, London, UK

${ }^{5}$ Department of Pathology, Fimlab Laboratories, Tampere University Hospital, Tampere, Finland ${ }^{6}$ Institute of Genetic Medicine, International Centre for Life, Newcastle University, Newcastle upon Tyne, UK ${ }^{7}$ Department of Neurology, Columbia University Medical Centre, New York, New York, USA

${ }^{8}$ Department of Neurology, Vasa Central Hospital, Vasa, Finland

Correspondence to Dr Johanna Palmio, Department of Neurology, Neuromuscular Research Centre, Tampere University and University Hospital, University of Tampere, Tampere 33014, Finland; johanna.palmio@uta.fi

Acknowledgements The Specialised Service for Rare Neuromuscular Diseases at Newcastle is funded under the NHS National Specialised services structure. The Newcastle Muscle team is part of the MRC Centre for Neuromuscular Diseases.

Contributors $\mathrm{KB}, \mathrm{BU}$ were involved in the study conception and design. $A E, A B, F N, K V, A V, S H, V S$, $M H, K B, B U$ were involved in the acquisition of data. $J P, A E, F N, K V, A V, S H, V S, P H, M H, K B, B U$ were involved in the analysis and interpretation of data. JP, $A B, V S, P H, M H, K B, B U$ were involved in the drafting and revision of the manuscript. $\mathrm{BU}$ obtained funding and involved in the study supervision.

Funding This work was supported by the Juselius Foundation as well as the Finnish Academy (BU).
Competing interests None declared.

\section{Patient consent Obtained.}

Ethics approval The study was carried out according to the ethical standards of the Helsinki declaration. All participants provided appropriate informed consent.

Provenance and peer review Not commissioned; externally peer reviewed.

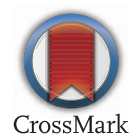

To cite Palmio J, Evilä A, Bashir A, et al. J Neurol Neurosurg Psychiatry 2016;87:448-450.

Received 28 August 2014

Revised 11 March 2015

Accepted 14 April 2015

Published Online First 7 May 2015

J Neurol Neurosurg Psychiatry 2016;87:448-450. doi:10.1136/jnnp-2014-309349

\section{REFERENCES}

1 Feit $H$, Silbergleit A, Schneider LB, et al. Vocal cord and pharyngeal weakness with autosomal dominant distal myopathy: clinical description and gene localization to 5q31. Am J Hum Genet 1998;63: 1732-42.

2 Senderek J, Garvey SM, Krieger M, et al. Autosomal-dominant distal myopathy associated with a recurrent missense mutation in the gene encoding the nuclear matrix protein, matrin 3. Am J Hum Genet 2009;84:511-18.

3 Müller TJ, Kraya T, Stoltenburg-Didinger G, et al. Phenotype of matrin-3-related distal myopathy in 16 German patients. Ann Neurol 2014;76: 669-80.

4 Yamashita S, Mori A, Nishida Y, et al. Clinicopathological features of the first Asian family having vocal cord and pharyngeal weakness with distal myopathy due to a MATR3 mutation. Neuropathol Appl Neurobiol 2014;41:391-8.

5 Johnson JO, Pioro EP, Boehringer $A$, et al. Mutations in the Matrin 3 gene cause familial amyotrophic lateral sclerosis. Nat Neurosci 2014;17:664-6.

6 Smith BN, Ticozzi N, Fallini C, et al. Exome-wide Rare Variant Analysis Identifies TUBA4A Mutations Associated with Familial ALS. Neuron 2014;84: 324-31.

7 Watts GD, Wymer J, Kovach MJ, et al. Inclusion body myopathy associated with Paget disease of bone and frontotemporal dementia is caused by mutant valosin-containing protein. Nat Genet 2004;36:377-81.

8 Johnson JO, Mandrioli J, Benatar M, et al. Exome sequencing reveals VCP mutations as a cause of familial ALS. Neuron 2010;68:857-64.

9 Kim HJ, Kim NC, Wang YD, et al. Mutations in prion-like domains in hnRNPA2B1 and hnRNPA1 cause multisystem proteinopathy and ALS. Nature 2013;495:467-73.

10 Bannwarth S, Ait-El-Mkadem S, Chaussenot A, et al. A mitochondrial origin for frontotemporal dementia and amyotrophic lateral sclerosis through CHCHD10 involvement. Brain 2014;137:2329-45.

11 Ajroud-Driss S, Fecto F, Ajroud K, et al. Mutation in the novel nuclear-encoded mitochondrial protein CHCHD10 in a family with autosomal dominant mitochondrial myopathy. Neurogenetics 2015;16: 1-9.

12 Nakayasu H, Berezney R. Nuclear matrins: identification of the major nuclear matrix proteins. Proc Natl Acad Sci USA 1991;88:10312-16. 
Re-evaluation of the phenotype caused by the common MATR3 p.Ser85Cys mutation in a new family

Johanna Palmio, Anni Evilä, Ayat Bashir, Fiona Norwood, Kati Viitaniemi, Anna Vihola, Sanna Huovinen, Volker Straub, Peter Hackman, Michio Hirano, Kate Bushby and Bjarne Udd

J Neurol Neurosurg Psychiatry 2016 87: 448-450 originally published online May 7, 2015

doi: 10.1136/jnnp-2014-309349

Updated information and services can be found at:

http://jnnp.bmj.com/content/87/4/448

\section{These include:}

References This article cites 12 articles, 2 of which you can access for free at: http://jnnp.bmj.com/content/87/4/448\#BIBL

Email alerting service

Receive free email alerts when new articles cite this article. Sign up in the box at the top right corner of the online article.

\section{Notes}

To request permissions go to:

http://group.bmj.com/group/rights-licensing/permissions

To order reprints go to:

http://journals.bmj.com/cgi/reprintform

To subscribe to BMJ go to:

http://group.bmj.com/subscribe/ 